\title{
Nonlocal nonlinear integrodifferential equations of fractional orders
}

\author{
Amar Debbouche ${ }^{1}$, Dumitru Baleanu ${ }^{2,3 *}$ and Ravi P Agarwal ${ }^{4}$
}

${ }^{\text {*Correspondence: }}$

dumitru@cankaya.edu.tr

${ }^{2}$ Department of Mathematics and

Computer Science, Cankaya

University, Ankara, Turkey

${ }^{3}$ Institute of Space Sciences, P.O. Box

MG-23, Magurele-Bucharest,

RO 76900, Romania

Full list of author information is

available at the end of the article

\begin{abstract}
In this paper, Schauder fixed point theorem, Gelfand-Shilov principles combined with semigroup theory are used to prove the existence of mild and strong solutions for nonlinear fractional integrodifferential equations of Sobolev type with nonlocal conditions in Banach spaces. To illustrate our abstract results, an example is given.

MSC: 35A05; 34G20; 34K05; 26A33
\end{abstract}

Keywords: fractional evolution equation; nonlocal condition; Schauder's fixed point theorem; uniformly continuous semigroup

\section{Introduction}

We are concerned with the nonlocal nonlinear fractional problem

$$
\begin{aligned}
& \frac{d^{\alpha}(B u(t))}{d t^{\alpha}}+A u(t)=f(t, W(t))+\int_{0}^{t} g(t, s, W(s)) d s, \\
& u(0)+\sum_{k=1}^{p} c_{k} u\left(t_{k}\right)=u_{0},
\end{aligned}
$$

where $\frac{d^{\alpha}}{d t^{\alpha}}, 0<\alpha \leq 1$ is the Riemann-Liouville fractional derivative, $0 \leq t_{1}<\cdots<t_{p} \leq a$, $c_{1}, \ldots, c_{p}$ are real numbers, $B$ and $A$ are linear closed operators with domains contained in a Banach space $X$ and ranges contained in a Banach space $Y, W(t)=\left(B_{1}(t) u(t), \ldots, B_{r}(t) u(t)\right)$, $\left\{B_{i}(t): i=1, \ldots, r, t \in I=[0, a]\right\}$ is a family of linear closed operators defined on dense sets $S_{1}, \ldots, S_{r} \supset D(A) \supset D(B)$ respectively in $X$ into $X, f: I \times X^{r} \rightarrow Y$ and $g: \Delta \times X^{r} \rightarrow Y$ are given abstract functions. Here $\Delta=\{(s, t): 0 \leq s \leq t \leq a\}$.

Fractional differential equations have attracted many authors $[1,6-8,21,24,25,30]$. This is mostly because it efficiently describes many phenomena arising in engineering, physics, economics and science. In fact, we can find several applications in viscoelasticity, electrochemistry, electromagnetic, etc. For example, Machado [22] gave a novel method for the design of fractional order digital controllers.

Following Gelfand and Shilov [20], we define the fractional integral of order $\alpha>0$ as

$$
I_{a}^{\alpha} f(t)=\frac{1}{\Gamma(\alpha)} \int_{a}^{t}(t-s)^{\alpha-1} f(s) d s,
$$

@ 2012 Debbouche et al.; licensee Springer. This is an Open Access article distributed under the terms of the Creative Commons Attribution License (http://creativecommons.org/licenses/by/2.0), which permits unrestricted use, distribution, and reproduction in any medium, provided the original work is properly cited. 
also, the (Riemann-Liouville) fractional derivative of the function $f$ of order $0<\alpha<1$ as

$$
{ }_{a} D_{t}^{\alpha} f(t)=\frac{1}{\Gamma(1-\alpha)} \frac{d}{d t} \int_{a}^{t}(t-s)^{-\alpha} f(s) d s,
$$

where $f$ is an abstract continuous function on the interval $[a, b]$ and $\Gamma(\alpha)$ is the Gamma function, see also [14, 24].

The existence results to evolution equations with nonlocal conditions in a Banach space was studied first by Byszewski $[9,10]$; subsequently, many authors were pointed to the same field, see for instance [2-4, 11-13, 19, 28].

Deng [15] indicated that using the nonlocal condition $u(0)+h(u)=u_{0}$ to describe, for instance, the diffusion phenomenon of a small amount of gas in a transparent tube can give a better result than using the usual local Cauchy problem $u(0)=u_{0}$. Let us observe also that since Deng's papers, the function $h$ is considered

$$
h(u)=\sum_{k=1}^{p} c_{k} u\left(t_{k}\right),
$$

where $c_{k}, k=1,2, \ldots, p$ are given constants and $0 \leq t_{1}<\cdots<t_{p} \leq a$.

However, among the previous research on nonlocal Cauchy problems, few authors have been concerned with mild solutions of fractional semilinear differential equations [23].

Recently, many authors have extended this work to various kinds of nonlinear evolution equations $[2,3,5,11,12,18]$. Balachandran and Uchiyama [3] proved the existence of mild and strong solutions of a nonlinear integrodifferential equation of Sobolev type with nonlocal condition.

In this paper, motivated by $[3,13,17,19]$, we use Schauder fixed point theorem and the semigroup theory to investigate the existence and uniqueness of mild and strong solutions for nonlinear fractional integrodifferential equations of Sobolev type with nonlocal conditions in Banach spaces, the solutions were obtained by using Gelfand-Shilov approach in fractional calculus and are given in terms of some probability density functions such that their Laplace transforms are indicated [17].

Our paper is organized as follows. Section 2 is devoted to the review of some essential results. In Section 3, we state and prove our main results; the last section deals with giving an example to illustrate the abstract results.

\section{Preliminary results}

In this section, we mention some results obtained by Balachandran [3], El-Borai [19] and Pazy [26], which will be used to get our new results. Let $X$ and $Y$ be Banach spaces with norm $|\cdot|$ and $\|\cdot\|$ respectively. The operator $B: D(B) \subset X \rightarrow Y$ satisfies the following hypotheses:

$\left(\mathrm{H}_{1}\right) \quad B$ is bijective,

$\left(\mathrm{H}_{2}\right) B^{-1}: Y \rightarrow D(B)$ is compact.

The above fact and the closed graph theorem imply the boundedness of the linear operator $A B^{-1}: Y \rightarrow Y$. Further $E=-A B^{-1}$ generates a uniformly continuous semigroup $Q(t), t \geq 0$ such that $\max _{t \in I}\|Q(t)\| \leq K, Q(t) h \in D(A),\|E Q(t) h\| \leq \frac{K}{t}\|h\|$ for every $h \in X$ and all $t \in$ $(0, a]$, see [29]. 
Let $\lambda=\left\|B^{-1}\right\|, c=\sum_{k=1}^{p}\left|c_{k}\right|$ and $\Lambda_{\tau}=\left\{\left(u_{1}, \ldots, u_{r}\right): u_{i} \in X, \sum_{i=1}^{r}\left|u_{i}\right| \leq \tau\right\}$.

It is supposed that

$\left(\mathrm{H}_{3}\right) f$ and $g$ are continuous in $t$ on $I, \Delta$ respectively, and there exist constants $M_{1}, M_{2}>0$ such that $\|f(t, W)\| \leq M_{1},\|g(t, s, W)\| \leq M_{2}$ for all $t \in I,(s, t) \in \Delta$ and $W \in \Lambda_{\tau}$.

Definition 2.1 By a strong solution of the nonlocal Cauchy problem (1.1), (1.2), we mean a function $u$ with values in $X$ such that

(i) $u$ is a continuous function in $t \in I$ and $u(t) \in D(A)$,

(ii) $\frac{d^{\alpha} u}{d t^{\alpha}}$ exists and is continuous on $(0, a], 0<\alpha<1$, and $u$ satisfies (1.1) on $(0, a]$ and (1.2).

Remark 2.1 Let us take in the considered problem $B$ is the identity, the inhomogeneous part is equal to an abstract continuous function $f(t)$, and the nonlocal condition is reduced to the initial condition $u(0)=u_{0}$, i.e.,

$$
\begin{aligned}
& D_{t}^{\alpha} u(t)+A u(t)=f(t), \\
& u(0)=u_{0} .
\end{aligned}
$$

According to El-Borai [17-19], we first apply the fractional integral on both sides of (2.1) and then using (2.2), we apply the Laplace transform on the new integral equations by considering a suitable one-sided stable probability density whose Laplace transform is given. Hence we can conclude that a solution of the problem (2.1)-(2.2) can be formally represented by

$$
u(t)=\int_{0}^{\infty} \zeta_{\alpha}(\theta) Q\left(t^{\alpha} \theta\right) u_{0} d \theta+\alpha \int_{0}^{t} \int_{0}^{\infty} \theta(t-s)^{\alpha-1} \zeta_{\alpha}(\theta) Q\left((t-s)^{\alpha} \theta\right) f(s) d \theta d s,
$$

where $\zeta_{\alpha}$ is a probability density function defined on $(0, \infty)$ such that its Laplace transform is given by

$$
\int_{0}^{\infty} e^{-\theta x} \zeta_{\alpha}(\theta) d \theta=\sum_{j=0}^{\infty} \frac{(-x)^{j}}{\Gamma(1+\alpha j)}, \quad 0<\alpha \leq 1, x>0 .
$$

For more details, we refer to Zhou et al. [27, 31], see also [14,16].

Using Gelfand-Shilov principle [20], it is suitable to rewrite (1.1), (1.2) in the form

$$
\begin{aligned}
B u(t)= & B u(0)+\frac{1}{\Gamma(\alpha)} \int_{0}^{t}(t-\eta)^{\alpha-1} \\
& \times\left[-A u(\eta)+f(\eta, W(\eta))+\int_{0}^{\eta} g(\eta, s, W(s)) d s\right] d \eta,
\end{aligned}
$$

where $\Gamma(\alpha)$ is the Gamma function.

According to [17-19], the equation (2.4) is equivalent to the integral equation

$$
B u(t)=\Psi(t) B u(0)+\int_{0}^{t} \Phi(t-\eta)\left[f(\eta, W(\eta))+\int_{0}^{\eta} g(\eta, s, W(s)) d s\right] d \eta, \quad t>0,
$$


where

$$
\begin{aligned}
& \Psi(t)=\int_{0}^{\infty} \zeta_{\alpha}(\theta) Q\left(t^{\alpha} \theta\right) d \theta, \\
& \Phi(t)=\alpha \int_{0}^{\infty} \theta t^{\alpha-1} \zeta_{\alpha}(\theta) Q\left(t^{\alpha} \theta\right) d \theta .
\end{aligned}
$$

It is assumed that there exists an operator $\psi$ on $D(\psi)=X$ given by the formula

$$
\psi=\left[I+\sum_{k=1}^{p} c_{k} B^{-1} \Psi\left(t_{k}\right) B\right]^{-1},
$$

satisfying $\psi u_{0} \in D(B)$ and for $k=1, \ldots, p$

$$
\psi \int_{0}^{t_{k}} B^{-1} \Phi\left(t_{k}-\eta\right)\left[f(\eta, W(\eta))+\int_{0}^{\eta} g(\eta, s, W(s)) d s\right] d \eta \in D(B)
$$

also

$\left(\mathrm{H}_{4}\right) K \lambda\left\|B \psi u_{0}\right\|\left[\lambda^{2} K^{2} c a^{\alpha}\|B \psi\|+\lambda K a^{\alpha}\right]\left(M_{1}+a M_{2}\right) \leq \tau$.

Further we assume

$\left(\mathrm{H}_{5}\right)$ There is a number $\gamma \in(0,1)$ such that

$$
\left\|B_{i}\left(t_{2}\right) Q\left(t_{1}\right) h\right\| \leq \frac{K_{1}}{t_{1}^{\gamma}}\|h\|
$$

where $t_{1} \in(0, a], t_{2} \in I, h \in X$ and $K_{1}$ is a positive constant, $i=1, \ldots, r$.

$\left(\mathrm{H}_{6}\right)$ The functions $B_{1}(t) h, \ldots, B_{r}(t) h$ are uniformly Hölder continuous in $t \in I$ for every element $h$ in $\bigcap_{i} S_{i}$.

Suppose that $\{Q(t)\}$ is a $C_{0}$-semigroup of operators on $X$ such that $\left\|B^{-1} Q\left(t_{k}\right) B\right\| \leq C e^{-\delta t_{k}}$, where $\delta$ is a positive constant and $C \geq 1$. Noting that $\int_{0}^{\infty} \zeta_{\alpha}(\theta) d \theta=1$ (see [14, p.4]).

If $\sum_{k=1}^{p}\left|c_{k}\right| e^{-\delta t_{k}}<\frac{1}{C}$, then $\left\|\sum_{k=1}^{p} c_{k} B^{-1} \psi\left(t_{k}\right) B\right\|<1$, which achieves that $\psi$ exists on $X$.

\section{Main results}

The following is different from $[3,19,26]$ and represents the new result.

Lemma 3.1 If $u$ is a continuous solution of (2.5), then $u$ satisfies the integral equation

$$
\begin{aligned}
u(t)= & B^{-1} \Psi(t) B \psi u_{0} \\
& -\sum_{k=1}^{p} c_{k} B^{-1} \Psi(t) B \psi \\
& \times \int_{0}^{t_{k}} B^{-1} \Phi\left(t_{k}-s\right)\left[f(s, W(s))+\int_{0}^{s} g(s, \eta, W(\eta)) d \eta\right] d s \\
& +\int_{0}^{t} B^{-1} \Phi(t-s)\left[f(s, W(s))+\int_{0}^{s} g(s, \eta, W(\eta)) d \eta\right] d s .
\end{aligned}
$$


Debbouche et al. Boundary Value Problems 2012, 2012:78

Page 5 of 10

Proof Using (2.5) and (1.2), we get

$$
\begin{aligned}
\sum_{k=1}^{p} c_{k} B u\left(t_{k}\right)= & \sum_{k=1}^{p} c_{k} \Psi\left(t_{k}\right) B u_{0}-\sum_{k=1}^{p} c_{k} \Psi\left(t_{k}\right) B \sum_{k=1}^{p} c_{k} u\left(t_{k}\right) \\
& +\sum_{k=1}^{p} c_{k} \int_{0}^{t_{k}} \Phi\left(t_{k}-s\right)\left[f(s, W(s))+\int_{0}^{s} g(s, \eta, W(\eta)) d \eta\right] d s .
\end{aligned}
$$

Then

$$
\begin{aligned}
\sum_{k=1}^{p} c_{k} u\left(t_{k}\right)\left[I+\sum_{k=1}^{p} c_{k} B^{-1} \Psi\left(t_{k}\right) B\right]= & \sum_{k=1}^{p} c_{k} B^{-1} \Psi\left(t_{k}\right) B u_{0}+\sum_{k=1}^{p} c_{k} \int_{0}^{t_{k}} B^{-1} \Phi\left(t_{k}-s\right) \\
& \times\left[f(s, W(s))+\int_{0}^{s} g(s, \eta, W(\eta)) d \eta\right] d s .
\end{aligned}
$$

Thus

$$
\begin{aligned}
\Psi(t) B u(0)= & \Psi(t)\left[B u_{0}-\sum_{k=1}^{p} c_{k} B u\left(t_{k}\right)\right] \\
= & \Psi(t) B u_{0}-\Psi(t) B \psi \sum_{k=1}^{p} c_{k} B^{-1} \Psi\left(t_{k}\right) B u_{0} \\
& -\Psi(t) B \psi \sum_{k=1}^{p} c_{k} \int_{0}^{t_{k}} B^{-1} \Phi\left(t_{k}-s\right)\left[f(s, W(s))+\int_{0}^{s} g(s, \eta, W(\eta)) d \eta\right] d s \\
= & \Psi(t) B \psi u_{0}\left[\psi^{-1}-\sum_{k=1}^{p} c_{k} B^{-1} \Psi\left(t_{k}\right) B\right] \\
& -\Psi(t) B \psi \sum_{k=1}^{p} c_{k} \int_{0}^{t_{k}} B^{-1} \Phi\left(t_{k}-s\right)\left[f(s, W(s))+\int_{0}^{s} g(s, \eta, W(\eta)) d \eta\right] d s .
\end{aligned}
$$

Hence the required result.

Definition 3.1 A continuous solution of the integral equation (3.1) is called a mild solatimon of the nonlocal problem (1.1), (1.2) on $I$.

Theorem 3.2 If the assumptions $\left(H_{1}\right) \sim\left(H_{4}\right)$ hold and $W(t)=u(t)$, then the problem (1.1), (1.2) has a mild solution on I.

Proof Let $Z=C(I, X)$ and $Z_{0}=\left\{u \in Z: u(t) \in \Lambda_{\tau}, t \in I\right\}$. It is easy to see that $Z_{0}$ is a bounded closed convex subset of $Z$. We define a mapping $\varphi: Z_{0} \rightarrow Z_{0}$ by

$$
\begin{aligned}
(\varphi u)(t)= & B^{-1} \Psi(t) B \psi u_{0} \\
& -\sum_{k=1}^{p} c_{k} B^{-1} \Psi(t) B \psi \int_{0}^{t_{k}} B^{-1} \Phi\left(t_{k}-s\right)\left[f(s, W(s))+\int_{0}^{s} g(s, \eta, W(\eta)) d \eta\right] d s \\
& +\int_{0}^{t} B^{-1} \Phi(t-s)\left[f(s, W(s))+\int_{0}^{s} g(s, \eta, W(\eta)) d \eta\right] d s, \quad t \in I .
\end{aligned}
$$


Noting also that $\int_{0}^{\infty} \theta \zeta_{\alpha}(\theta) d \theta=1$ (see [14, p.4]), we have

$$
\begin{aligned}
\|(\varphi u)(t)\| \leq & K \lambda\left\|B \psi u_{0}\right\|+\left(M_{1}+a M_{2}\right) \lambda^{2} K^{2} c a^{\alpha}\|B \psi\| \\
& +\left(M_{1}+a M_{2}\right) \lambda K a^{\alpha} \leq \tau .
\end{aligned}
$$

We deduce that $\varphi$ is continuous and maps $Z_{0}$ into itself. Moreover, $\varphi$ maps $Z_{0}$ into a precompact subset of $Z_{0}$. Note that the set $Z_{0}(t)=\left\{(\varphi u)(t): u \in Z_{0}\right\}$ is precompact in $X$, for every fixed $t \in I$. We shall show that $\varphi\left(Z_{0}\right)=S=\left\{\varphi u: u \in Z_{0}\right\}$ is an equicontinuous family of functions. For $0<s<t$, we have

$$
\begin{aligned}
& \|(\varphi u)(t)-(\varphi u)(s)\| \\
& \leq \quad\left[\lambda\left\|B \psi u_{0}\right\|+c \lambda^{2} K a^{\alpha}\left(M_{1}+a M_{2}\right)\|B \psi\|\right]\|\Psi(t)-\Psi(s)\| \\
& \quad+\lambda\left(M_{1}+a M_{2}\right) \int_{s}^{t}\|\Phi(t-\eta)\| d \eta \\
& \quad+\lambda\left(M_{1}+a M_{2}\right) \int_{0}^{s}\|\Phi(t-\eta)-\Phi(s-\eta)\| d \eta .
\end{aligned}
$$

The right-hand side of the above inequality is independent of $u \in Z_{0}$ and tends to zero as $s \rightarrow t$ as a consequence of the continuity of $\Psi(t)$ and $\Phi(t)$ in the uniform operator topology for $t>0$. It is clear that $S$ is bounded in $Z$. Thus by Arzela-Ascoli's theorem, $S$ is precompact. Hence by the Schauder fixed point theorem, $\varphi$ has a fixed point in $Z_{0}$ and any fixed point of $\varphi$ is a mild solution of (1.1), (1.2) on $I$ such that $u(t) \in X$ for all $t \in I$.

\section{Theorem 3.3 Assume that}

(i) Conditions $\left(H_{1}\right) \sim\left(H_{6}\right)$ hold,

(ii) $Y$ is a reflexive Banach space with norm $\|\cdot\|$,

(iii) there are numbers $L_{1}, L_{2}>0$ and $p, q \in(0,1]$ such that

$$
\begin{aligned}
& \left\|f\left(t_{1}, W\right)-f\left(t_{2}, W^{*}\right)\right\| \leq L_{1}\left(\left|t_{1}-t_{2}\right|^{p}+\sum_{i=1}^{r}\left|w_{i}-w_{i}^{*}\right|\right), \\
& \left\|g\left(s_{1}, \eta, W\right)-g\left(s_{2}, \eta, W\right)\right\| \leq L_{2}\left|s_{1}-s_{2}\right|^{q}
\end{aligned}
$$

for all $t_{1}, t_{2} \in I,\left(s_{1}, \eta\right),\left(s_{2}, \eta\right) \in \Delta$ and all $W, W^{*} \in \Lambda_{\tau}$, where $w_{i}=B_{i} u$ and $w_{i}^{*}=B_{i} u^{*}$. Then the problem (1.1), (1.2) has a unique strong solution on $I$.

Proof Applying Theorem 3.2, the problem (1.1), (1.2) has a mild solution $u \in C\left(I, \Lambda_{\tau}\right)$. Now, we shall show that $u$ is a unique strong solution of the considered problem on $I$.

According to $\left(\mathrm{H}_{6}\right), \sum_{i=1}^{r}\left|w_{i}-w_{i}^{*}\right|$ is uniformly Hölder continuous in $t \in I$ for every element $u$ in $\bigcap_{i} S_{i}$ combined with (iii), which implies that $t \rightarrow f(t, W(t))$ and $t \rightarrow$ $\int_{0}^{t} g(t, s, W(s)) d s$ are uniformly Hölder continuous on $I$.

Set

$$
V(t)=f(t, W(t))+\int_{0}^{t} g(t, s, W(s)) d s
$$


From (3.1), the solution $u$ of the considered problem can be written in the form

$$
\begin{aligned}
u(t)= & B^{-1} \Psi(t) B \psi u_{0}-B^{-1} \Psi(t) B \psi \sum_{k=1}^{p} c_{k} \int_{0}^{t_{k}} B^{-1} \Phi\left(t_{k}-s\right) V(s) d s \\
& +\int_{0}^{t} B^{-1} \Phi(t-s) V(s) d s .
\end{aligned}
$$

Noting that $\Psi$ and $\psi$ are bounded, using our assumptions, we observe that there exists a unique function $V \in C(I, X)$ which satisfies the equation

$$
\frac{d^{\alpha}(B u(t))}{d t^{\alpha}}+A u(t)=V(t)
$$

Also as in [19, p.409], we deduce that

$$
\int_{0}^{t} B^{-1} \Phi(t-s) V(s) d s \in D(E)
$$

for all $t \in I$ and $\psi u_{0} \in D(E)$. It follows that $u(t) \in D(E)$ for all $t \in I$.

\section{Example}

Consider the nonlinear integro-partial differential equation of fractional order

$$
\begin{gathered}
\frac{\partial^{\alpha}\left[\sum_{|q| \leq 2 m} b_{q}(x) D_{x}^{q} u(x, t)\right]}{\partial t^{\alpha}}+\sum_{|q| \leq 2 m} a_{q}(x) D_{x}^{q} u(x, t) \\
=F(x, t, W)+\int_{0}^{t} G(x, t, s, W(s)) d s,
\end{gathered}
$$

with nonlocal condition

$$
u(x, 0)+\sum_{k=1}^{p} c_{k} u\left(x, t_{k}\right)=g(x),
$$

where $0<\alpha \leq 1,0 \leq t_{1}<\cdots<t_{p} \leq a, x \in R^{n}, D_{x}^{q}=D_{x_{1}}^{q_{1}} \cdots D_{x_{n}}^{q_{n}}, D_{x_{i}}=\frac{\partial}{\partial x_{i}}, q=\left(q_{1}, \ldots, q_{n}\right)$ is an $n$-dimensional multi-index, $|q|=q_{1}+\cdots+q_{n}, W=\left(w_{1}, \ldots, w_{r}\right)$,

$$
w_{i}(x, t)=\sum_{|q| \leq 2 m-1} b_{q_{i}}(x, t) D_{x}^{q} u(x, t)+\int_{\Omega} \sum_{|q| \leq 2 m-1} c_{q_{i}}(x, t) D_{y}^{q} u(y, t) d y,
$$

and $\Omega$ is an open subset of $R^{n}$. Let $L_{2}\left(R^{n}\right)$ be the set of all square integrable functions on $R^{n}$. We denote by $C^{m}\left(R^{n}\right)$ the set of all continuous real-valued functions defined on $R^{n}$ which have continuous partial derivatives of order less than or equal to $m$. By $C_{0}^{m}\left(R^{n}\right)$ we denote the set of all functions $f \in C^{m}\left(R^{n}\right)$ with compact supports. Let $H^{m}\left(R^{n}\right)$ be the completion of $C_{0}^{m}\left(R^{n}\right)$ with respect to the norm

$$
\|f\|_{m}^{2}=\sum_{|q| \leq m} \int_{R^{n}}\left|D_{x}^{q} f(x)\right|^{2} d x
$$


It is supposed that

(i) The operator $E=-\sum_{|q|=2 m} e_{q}(x) D_{x}^{q}$ is uniformly elliptic on $R^{n}$. In other words, all the coefficients $e_{q},|q|=2 m$ are continuous and bounded on $R^{n}$, and there is a positive number $c$ such that

$$
(-1)^{m+1} \sum_{|q|=2 m} e_{q}(x) \xi^{q} \geq c|\xi|^{2 m}
$$

for all $x \in R^{n}$ and all $\xi \neq 0, \xi \in R^{n}$, where $e_{q}=a_{q} b_{q}^{-1}, \xi^{q}=\xi_{1}^{q_{1}} \cdots \xi_{n}^{q_{n}}$ and $|\xi|^{2}=\xi_{1}^{2}+\cdots+\xi_{n}^{2}$.

(ii) All the coefficients $e_{q},|q|=2 m$, satisfy a uniform Hölder condition on $R^{n}$. Under these conditions, the operator $E$ with the domain of definition $D(E)=H^{2 m}\left(R^{n}\right)$ generates an analytic semigroup $Q(t)$ defined on $L_{2}\left(R^{n}\right)$, and it is well known that $H^{2 m}\left(R^{n}\right)$ is dense in $Y=L_{2}\left(R^{n}\right)$, see $[17, \mathrm{p} .438]$.

Lemma 4.1 The solution representation of (4.1), (4.2) can be written explicitly.

Proof Let $\left\{E_{q}(x):|q| \leq 2 m\right\}$ be a family of deterministic square matrices of order $k$ and let $L(x, D)=\left\{E_{q}(x):|q|=2 m\right\}$. We assume that

$$
\operatorname{det}\left\{(-1)^{m} L(x, \sigma)-\lambda I\right\}=0
$$

has roots which satisfy the inequality $\operatorname{Re} \lambda<-\delta, \delta>0$ for all $x \in R^{n}$ and for any real vector $\sigma, \sigma_{1}^{2}+\cdots+\sigma_{n}^{2}=1$. If $\Theta$ is a matrix of order $m \times n$, then we introduce $|\Theta|=\sum_{i, j}\left|b_{i j}\right|$.

It is well known that there exists a fundamental matrix solution $Z(x, y, t)$ which satisfies the system

$$
\begin{aligned}
& \frac{\partial u(x, t)}{\partial t}=L(x, D) u(x, t), \quad t>0, x \in R^{n}, \\
& u\left(x, t_{0}\right)+\sum_{i=1}^{N} c_{i} u\left(x, t_{i}\right)=g(x) .
\end{aligned}
$$

This fundamental matrix also satisfies the inequality

$$
\left|D_{x}^{q} Z(x, y, t)\right| \leq K_{1} t^{-\rho_{1}} \exp \left(-K_{2} \rho_{2}\right)
$$

where $|q| \leq 2 m, \rho_{1}=-\frac{n+|q|}{2 m}, \rho_{2}=\sum_{i=1}^{n}\left|x_{i}-y_{i}\right|^{\lambda} t^{-\frac{1}{2 m-1}}, \lambda=\frac{2 m}{2 m-1}$ and $K_{1}, K_{2}$ are positive constants. From [13, p.58], if the nonlocal function $g(x)$ is an element in Hilbert space $H^{2 m}\left(R^{n}\right)$, then we can write

$$
Q(t) g(x)=\int_{R^{n}} Z(x-y, t) g(y) d y .
$$

It can be proved that

$$
\left\|D_{x}^{q} Q(t) g\right\| \leq \frac{M}{t^{\beta}}\|g\|
$$

where $0<\beta<1, M$ is a positive constant, $|q| \leq 2 m-1, t>0$ and $\|g\|^{2}=\int_{R^{n}} g^{2}(x) d x$. 
([17]) The nonlocal Cauchy problems (4.1), (4.2) are equivalent to the integral equation

$$
\begin{aligned}
u(x, t)= & \int_{0}^{\infty} \int_{R^{n}} \zeta_{\alpha}(\theta) Q\left(x-\xi, t^{\alpha} \theta\right) u(\xi, 0) d \xi d \theta \\
& +\alpha \int_{0}^{t} \int_{0}^{\infty} \int_{R^{n}} \theta(t-\eta)^{\alpha-1} \zeta_{\alpha}(\theta) Q\left(x-\xi,(t-\eta)^{\alpha} \theta\right) \\
& \times\left[F(\xi, \eta, W)+\int_{0}^{\eta} G(\xi, \eta, s, W(s)) d s\right] d \xi d \theta d \eta
\end{aligned}
$$

where the explicit form of $Q$ is given by

$$
Q(x, t)=\frac{e^{-|x|^{2} / 4 t}}{(\sqrt{4 \pi t})^{n}}
$$

$|x|^{2}=x_{1}^{2}+x_{2}^{2}+\cdots+x_{n}^{2}$. Applying Theorem 3.2, we achieve the proof of the existence of mild solutions of the problems (4.1), (4.2). In addition, if the operators $F$ and $G$ satisfy the following:

(iii) There are numbers $L_{1}, L_{2} \geq 0$ and $0<p, q \leq 1$ such that

$$
\sum_{|q| \leq 2 m-1} \int_{R^{n}}\left|F\left(x, t, D_{x}^{q} W\right)-F\left(x, s, D_{x}^{q} W^{*}\right)\right|^{2} d x \leq L_{1}\left(|t-s|^{p}+\sum_{i=1}^{r}\left|w_{i}-w_{i}^{*}\right|^{2} d x\right)
$$

and

$$
\sum_{|q| \leq 2 m-1} \int_{R^{n}}\left|G\left(x, t, \eta, D_{x}^{q} W\right)-G\left(x, s, \eta, D_{x}^{q} W\right)\right|^{2} d x \leq L_{2}|t-s|^{q}
$$

for all $t, s \in I,(t, \eta),(s, \eta) \in \Delta, W, W^{*} \in \Lambda_{\tau}$ and all $x \in R^{n}$. Then applying Theorem 3.3, we deduce that (4.1), (4.2) has a unique strong solution.

\section{Conclusion}

In this article, a new solution representation for Sobolev type fractional evolution equation has been proved using Deng's nonlocal condition, a suitable explicit form of the semigroup has been discussed. Moreover, the existence result of mild solutions for nonlinear fractional integrodifferential equations of Sobolev type with nonlocal conditions in Banach spaces has been established by using Arzela-Ascoli's theorem and Schauder fixed point theorem. Further, the uniformly Hölder continuous condition has been applied for the existence of strong solution.

'Department of Mathematics, Faculty of Science, Guelma University, Guelma, Algeria. ${ }^{2}$ Department of Mathematics and Computer Science, Cankaya University, Ankara, Turkey. ${ }^{3}$ Institute of Space Sciences, P.O. Box MG-23, Magurele-Bucharest, RO 76900, Romania. ${ }^{4}$ Department of Mathematics, Texas A\&M University, Kingsville, TX 78363, USA. 


\section{Acknowledgements}

The authors would like to thank the referees for their valuable comments and remarks.

\section{Received: 11 May 2012 Accepted: 9 July 2012 Published: 24 July 2012}

\section{References}

1. Agarwal, RP, Lakshmikanthama, V, Nieto, JJ: On the concept of solution for fractional differential equations with uncertainty. Nonlinear Anal. 72, 2859-2862 (2010)

2. Ahmad, B, Nieto, JJ: Existence of solutions for nonlocal boundary value problems of higher order nonlinear fractional differential equations. Abstr. Appl. Anal. 2009, Article ID 494720 (2009)

3. Balachandran, K, Uchiyama, K: Existence of solutions of nonlinear integrodifferential equations of Sobolev type with nonlocal condition in Banach spaces. Proc. Indian Acad. Sci. Math. Sci. 110(2), 225-232 (2000)

4. Balachandran, K, Samuel, FP: Existence of solutions for quasilinear delay integrodifferential equations with nonlocal conditions. Electron. J. Differ. Equ. 2009(6), 1-7 (2009)

5. Balachandran, K, Kumar, RR: Existence of solutions of integrodifferential evolution equations with time varying delays. Appl. Math. E-Notes 7, 1-8 (2007)

6. Baleanu, D, Mustafa, OG: On the global existence of solutions to a class of fractional differential equations. Comput. Math. Appl. 59(5), 1835-1841 (2010)

7. Baleanu, D, Mustafa, OG, Agarwal, RP: Asymptotically linear solutions for some linear fractional differential equations. Abstr. Appl. Anal. 2010, Article ID 865139 (2010)

8. Belmekki, M, Benchohra, M: Existence results for fractional order semilinear functional differential equations with nondense domain. Nonlinear Anal. 72, 925-932 (2010)

9. Byszewski, L: Theorems about the existence and uniqueness of solutions of a semilinear evolution nonlocal Cauchy problem. J. Math. Anal. Appl. 162, 494-505 (1991)

10. Byszewski, L: Applications of properties of the right hand sides of evolution equations to an investigation of nonlocal evolution problems. Nonlinear Anal. 33, 413-426 (1998)

11. Debbouche, A, Baleanu, D: Controllability of fractional evolution nonlocal impulsive quasilinear delay integro-differential systems. Comput. Math. Appl. 62, 1442-1450 (2011)

12. Debbouche, A: Fractional nonlocal impulsive quasilinear multi-delay integro-differential systems. Adv. Differ. Equ. 5, 1-10 (2011)

13. Debbouche, A: Fractional evolution integro-differential systems with nonlocal conditions. Adv. Dyn. Syst. Appl. 5(1), 49-60 (2010)

14. Debbouche, A, El-Borai, MM: Weak almost periodic and optimal mild solutions of fractional evolution equations. Electron. J. Differ. Equ. 2009(46), 1-8 (2009)

15. Deng, K: Exponential decay of solutions of semilinear parabolic equations with nonlocal initial conditions. J. Math Anal. Appl. 179, 630-637 (1993)

16. El-Borai, MM, Debbouche, A: On some fractional integro-differential equations with analytic semigroups. Int. J. Contemp. Math. Sci. 4(28), 1361-1371 (2009)

17. El-Borai, MM: Some probability densities and fundamental solutions of fractional evolution equations. Chaos Solitons Fractals 14(3), 433-440 (2002)

18. El-Borai, MM: Semigroups and some nonlinear fractional differential equations. Appl. Math. Comput. 149(3), 823-83 (2004)

19. El-Borai, MM: On some fractional evolution equations with nonlocal conditions. Int. J. Pure Appl. Math. 24(3), 405-413 (2005)

20. Gelfand, IM, Shilov, GE: Generalized Functions, vol. 1. Nauka, Moscow (1959)

21. Li, F: Mild solutions for fractional differential equations with nonlocal conditions. Adv. Differ. Equ. 2010, Article ID $287861(2010)$

22. Machado, JAT: Analysis and design of fractional-order digital control systems. Syst. Anal. Model. Simul. 27(2-3), 107-122 (1997)

23. Mophou, GM, N'Guerekata, GM: Mild solutions for semilinear fractional differential equations. Electron. J. Differ. Equ. 2009(21), 1-9 (2009)

24. Podlubny, I: Fractional Differential Equations. Math Science and Eng., vol. 198. Academic Press, San Diego (1999)

25. Tatar, N-E: On a boundary controller of fractional type. Nonlinear Anal. 72, 3209-3215 (2010)

26. Pazy, A: Semigroups of Linear Operators and Applications to Partial Differential Equations. Springer, Berlin (1983)

27. Wang, JR, Zhou, Y: A class of fractional evolution equations and optimal controls. Nonlinear Anal., Real World Appl. $12,262-272(2011)$

28. Yan, Z: Controllability of semilinear integrodifferential systems with nonlocal conditions. Int. J. Comput. Appl. Math. 3(2), 221-236 (2007)

29. Zaidman, S: Abstract Differential Equations. Pitman, London (1979)

30. Zhao, Y, Sun, S, Han, Z, Li, Q: Positive solutions to boundary value problems of nonlinear fractional differential equations. Abstr. Appl. Anal. 2011, Article ID 390543 (2011)

31. Zhou, Y, Jiao, F: Nonlocal Cauchy problem for fractional evolution equations. Nonlinear Anal., Real World Appl. 11, $4465-4475(2010)$

doi:10.1186/1687-2770-2012-78

Cite this article as: Debbouche et al.: Nonlocal nonlinear integrodifferential equations of fractional orders. Boundary Value Problems 2012 2012:78. 